\title{
Síndrome de Currarino. Informe de un caso
}

\author{
Nathalia Vélez, MD², Luz Stella López, MD², Jorge Alberto Rivera, MD², \\ Abraham Chams Anturi, MD ${ }^{4}$, David Andrés Espinal, MD ${ }^{5}$
}

\section{RESUMEN}

El síndrome de Currarino es una enfermedad hereditaria rara. La forma completa se caracteriza por la presencia de una malformación ano-rectal (generalmente estenosis), masa presacra y defectos óseos del sacro. El signo más importante es la presencia de estreñimiento desde el nacimiento. Se presenta un paciente de 13 meses de edad que consultó por estreñimiento crónico severo en quien se encontró la presencia de hemisacro y meningocele anterior, ambos hallazgos compatibles con una forma leve del síndrome de Currarino. Se realizó un manejo conservador y se inició polietilenglicol oral (PEG®), con una excelente respuesta al tratamiento. Se hace la presentación del caso junto con la revisión de la literatura actual con el fin de dar a conocer al personal médico la presencia del síndrome, enfatizar en puntos importantes que generen sospecha de su presencia y las diferentes opciones terapéuticas para su manejo.

Palabras clave: Síndrome de Currarino; Anomalía sacra; Estreñimiento crónico; Lactante.

\section{Currarino's syndrome. A case report}

\section{SUMMARY}

The Currarino's syndrome is a rare inherited disorder. The complete form is characterized by the presence of ano-rectal malformation (usually stenosis), presacral mass and bone defects of the sacrum. The most important symptom is the presence of constipation since birth. A 13 month old patient with chronic and severe constipation is presented. Among the studies the presence of hemisacrum and anterior meningocele were found, both findings consistent with a mild form of Currarino's syndrome. A conservative management with polietilenglycol oral (PEG®), was initiated with an excellent response to treatment. The presentation of the case with a review of current literature is given to the medical staff in order to publicize the syndrome, as well as to emphasize key points that create suspicion of their presence and the different therapeutic options for its approach.

\section{Keywords: Currarino’s syndrome; Sacrum abnormalities; Chronic constipation; Infant.}

En 1926 se comunica por primera vez una asociación de defecto sacro, meningocele sacro-anterior y malformación ano-rectal (MAR). Después, en 1974, lo informan como tríada en una descripción de 21 casos de defectos presacros y 12 de estos enfermos adicionalmente presentaban una estenosis ano-rectal. En 1981 Currarino et al. ${ }^{1}$ describen la tríada como un síndrome completo formado por hemisacro o sacro en «cimitarra», malformación ano-rectal y masa presacra.
Debido a las observaciones realizadas en las que se evidencia que el compromiso del sacro ocurre siempre por debajo del nivel de S1, se sugiere que los hallazgos vistos en el síndrome de Currarino se producen por un error en la migración de la eminencia caudal pluripotencial, luego de la primera neurulación, en etapas muy tempranas del desarrollo embrionario. Estudios genéticos sugieren que el locus implicado con el desarrollo ano-rectal y sacro es el gen HLXB9 q36 en el

1. Pediatra, Universidad Pontificia Bolivariana, Medellín.e-mail:velezsanjuan@gmail.com

2. Pediatra, Universidad CES, Medellín. e-mail: lusafe4@une.net.co

3. Pediatra Gastroenterólogo-Nutriólogo, Hospital Pablo Tobón Uribe, Medellín. e-mail: jariverae@une.net.co

4. Cirujano Pediátrico, Hospital Pablo Tobón Uribe y Hospital Universitario San Vicente de Paul. Docente de Cirugía Pediátrica, Universidad de Antioquia, Medellín. e-mail: abrahamchams@hotmail.com

5. Jefe Departamento Pediatría, Hospital Pablo Tobón Uribe, Medellín. e-mail: davidandres@une.net.co Recibido para publicación marzo 27, 2008 Aceptado para publicación abril 14, 2009 


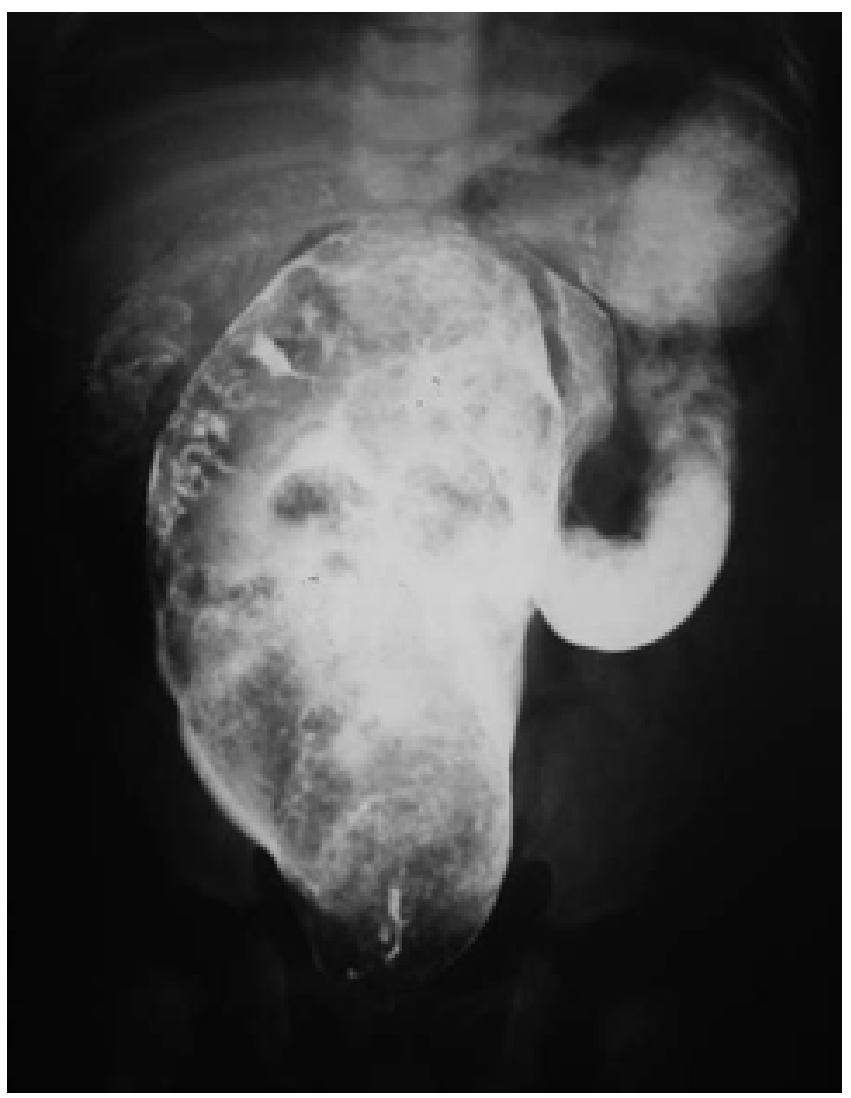

Figura 1. Radiografía colon por enema: Gran dilatación de la ampolla rectal sin zonas de transición

cromosoma 7. La real incidencia del síndrome de Currarino no se ha definido bien, pero desde el primer informe en 1926 se han descrito aproximadamente 250 casos con la tríada clínica completa consistente en defecto óseo sacro, malformación ano-rectal (MAR) y masa presacra. Se hace a continuación el informe de un caso en un lactante y la revisión de la literatura.

\section{CASO CLÍNICO}

Paciente masculino de 13 meses de edad, natural y residente en Armenia (Quindío). Valorado en la consulta de gastroenterología pediátrica del Hospital Pablo Tobón Uribe de Medellín por historia de estreñimiento crónico severo desde las 3 semanas de edad. A lo largo de su vida, había recibido manejo sintomático con enemas y laxantes logrando evacuar una o dos veces por semana. Al interrogatorio no hubo otros problemas gastrointestinales o urinarios, ni tampoco antecedentes médicos de importancia. En la historia familiar, el padre manifestó estreñimiento crónico presente desde la infancia.

Al examen físico hubo un peso de $9 \mathrm{~kg}$ y una talla de $74 \mathrm{~cm}$. El abdomen se observaba distendido y a la palpación se notó abundante cantidad de materia fecal en el marco cólico izquierdo. El resto del examen físico fue normal.

Entre los estudios hechos al paciente antes del momento de la consulta, había unas radiografías de colon por enema tomadas a los 3 meses de edad (Figura 1) en las que se visualizaba gran dilatación de la ampolla rectal sin zonas de transición y una biopsia de recto con presencia de plexos mientéricos submucosos e intramurales y filetes nerviosos en lámina propia.

El paciente se hospitaliza para tratamiento y estudio de su enfermedad para descartar la presencia de un componente orgánico que explique su estreñimiento crónico severo. Los estudios fueron:

- Cuadro hemático, ionograma, función renal y función hepática normales.

- Rx de columna lumbo-sacra: Gran distensión de asas del colon con abundante cantidad de materia fecal en su interior. Alteración en la morfología del sacro y el coxis sin lograrse visualizar adecuadamente por la presencia de gas intestinal (Figura 2).

- Ecografía abdominal total: Gran distensión de asas, no se evidenciaron alteraciones en morfología renal ni urinaria ni en otros sistemas.

- Manometría ano-rectal: Esfínter anal interno normal, presencia de reflejo recto-inhibitorio.

Ante la presencia de la anomalía en la morfología sacra según los rayos $\mathrm{X}$ se solicitó resonancia magnética simple y contrastada de columna lumbo-sacra (Figura 3) que informó cuerpos vertebrales lumbares normales, sacro en cimitarra con preservación de la morfología de S1 y S2, meningocele sacro anterior por debajo de S2, importante dilatación del colon distal y del recto con desplazamiento anterior y lateral izquierdo de la vejiga y cono medular a la altura de L2. Con estos hallazgos, se propuso el diagnóstico de síndrome de Currarino.

El niño fue evaluado por neurocirugía, cirugía pediátrica y gastropediatría y se acordó manejo conservador. Inicialmente se le desimpacta con enemas de fosfato de sodio y después se inició manejo con polietilenglicol oral (PEG ${ }^{\circledR}$ ) a dosis de 1.5 g/kg/día en 

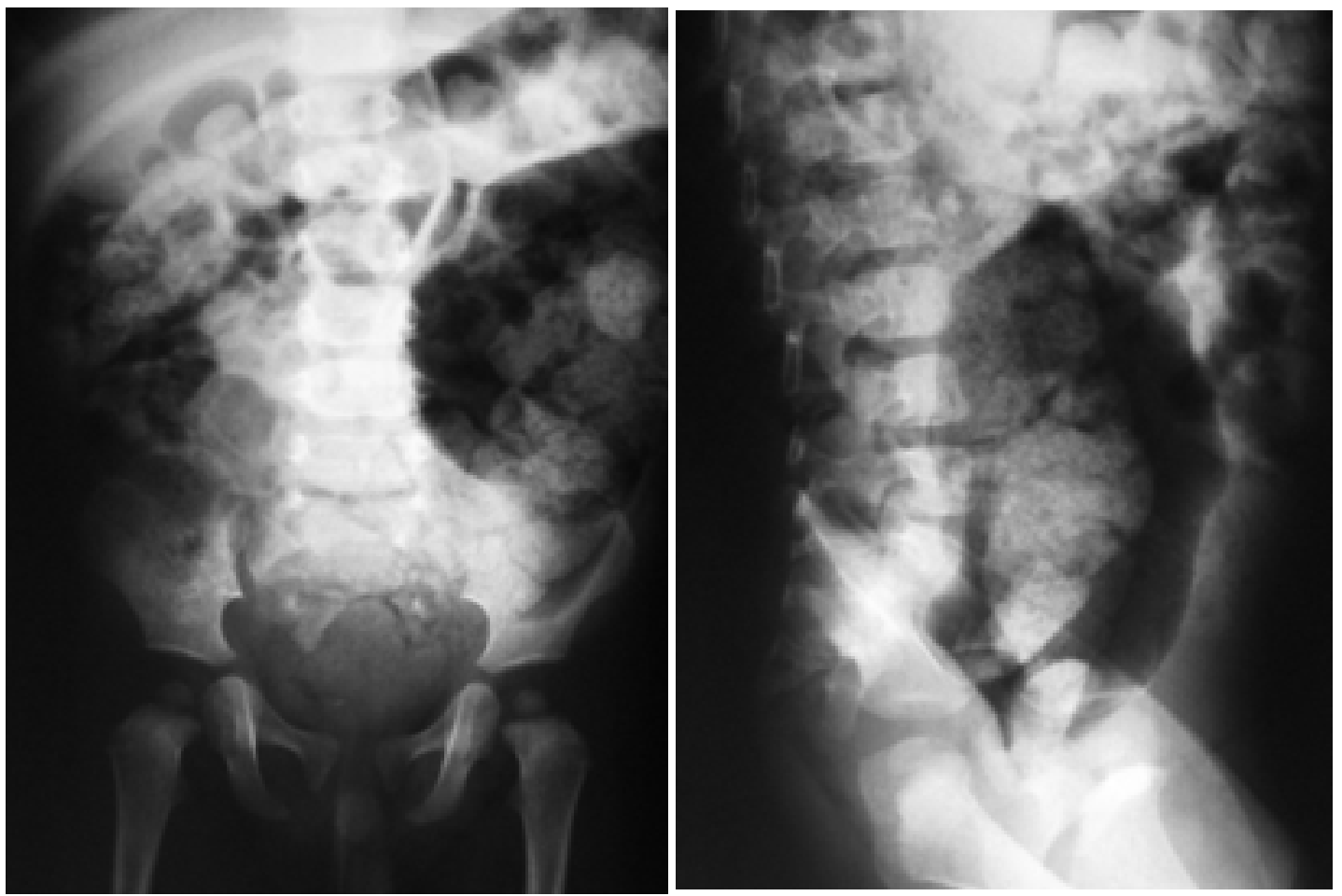

Figura 2. Radiografía AP y lateral de columna lumbo-sacra. Gran distensión del colon con abundante cantidad de materia fecal en su interior. Sacro con morfología anormal «sacro en cimitarra»
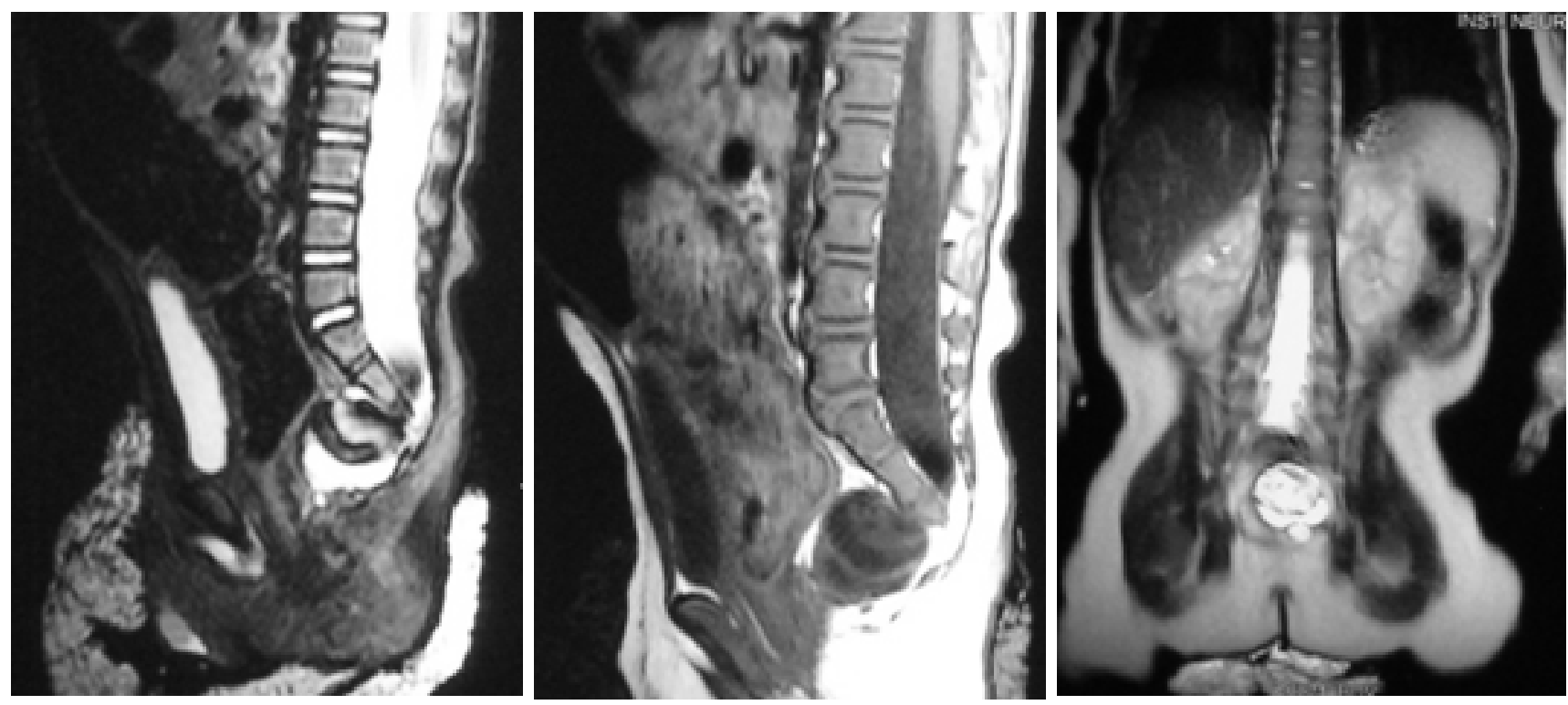

Figura 3. RM simple y contrastada de columna lumbo-sacra que demuestra meningocele anterior y sacro en cimitarra 
una sola toma diaria que da buena respuesta clínica, consistente en 1 ó 2 evacuaciones blandas al día, lo que se ha mantenido en el tiempo sin llegar a requerir aumento de la dosis ni adición de otro tipo de medicación laxante. Debido al hallazgo de estreñimiento crónico en el padre, se solicitó estudio genético al paciente y rayos $\mathrm{X}$ de columna lumbo-sacra al padre, pero, los resultados de estos exámenes continúan pendientes a la fecha.

\section{DISCUSIÓN}

El síndrome de Currarino es un desorden autosómico dominante con penetrancia variable ligado al gen HLXB9 en la región 7q36. Es característico de este síndrome una tríada clásica de hallazgos como son: el defecto óseo sacro, la malformación ano-rectal (MAR) y la masa presacra ${ }^{2}$.

El defecto óseo del sacro es el componente principal del síndrome que está presente en todos los casos y corresponde a la malformación sacra tipo IV o «sacro en cimitarra» donde se observa presencia de un hemisacro con preservación en la morfología de S1. El «sacro en cimitarra» es el hallazgo patognomónico del síndrome de Currarino.

El tipo de MAR más frecuentemente encontrado en el síndrome de Currarino es la MAR con fístula rectoperineal, sin embargo diferentes tipos de MAR (fístula rectouretral, fístula rectovestibular o rectoespinal) pueden estar presentes.

La masa presacra puede ser un meningocele anterior, un teratoma (generalmente benigno cuando se presenta en el contexto de este síndrome), un quiste dermoide, una duplicación rectal u otro tumor no común. Otros defectos del tubo neural o lipomas, son asociaciones que se pueden encontrar junto al meningocele anterior.

Adicionalmente, puede haber otras malformaciones asociadas como: malformaciones urológicas (doble uréter, doble riñón, reflujo vesicoureteral, riñón displásico o hipospadias), malformaciones genitales (útero bicorne, vagina septada, genitales ambiguos y clítoris bífido) y malformaciones en el sistema nervioso central (malformación de Arnold Chiari tipo I).

El síndrome de Currarino tiene una gran variabilidad en su fenotipo, muchos casos presentan un hemisacro asintomático sin otras anomalías, pero otros presentan los demás componentes de la tríada por lo que se ha propuesto la siguiente clasificación ${ }^{3}$ :

1. Completo: Se presenta con los 3 componentes de la tríada: hemisacro, MAR y masa presacra. El diagnóstico puede ser hecho desde el nacimiento.

2. Leve: Presencia del hemisacro y apenas uno de los otros dos hallazgos: MAR o masa presacra. En caso de MAR el diagnóstico puede ser hecho desde el nacimiento cuando hay MAR, sin embargo en el otro caso, el diagnóstico es más difícil y de manifestación tardía con el estreñimiento como síntoma principal.

3. Mínimo: Tan sólo el hemisacro está presente. El estreñimiento también es común.

Las principales complicaciones asociadas con este síndrome son: Estreñimiento crónico, sepsis de origen perineal, meningitis por enteropatógenos, abscesos anorectales, infección urinaria recurrente, dispareunia, degeneración maligna del teratoma presacro, aborto recurrente, compresión medular o dificultades para un parto vaginal en mujeres gestantes.

Aunque en la literatura hay pocos informes de casos, el rango de edad descrito para el diagnóstico oscila entre 1 y 65 años y el síntoma más común es el estreñimiento de aparición temprana y de difícil manejo para el cual se han postulado varias posibles etiologías como la estenosis anal, el efecto compresivo de la masa presacra o la asociación con enfermedad de Hirschprung u otras disganglionosis ${ }^{3}$. Los pacientes que presentan masa presacra pueden tener síntomas neurológicos, otros pueden aparecer con sepsis o meningitis por Escherichia coli (atribuible a contaminación fecal del LCR) y otros pueden presentarse con infección urinaria (por asociación con reflujo vesicoureteral), abscesos perianales, dolor lumbar o cefalea recurrente.

El tacto rectal puede o no evidenciar la masa presacra o la estenosis del canal recto-anal y debe estar seguido por la toma de una radiografía de pelvis en proyecciones AP y lateral para evaluar el índice sacro y la presencia de anomalías lumbosacras. La presencia de un «sacro en cimitarra» con preservación de la morfología de S1, se constituye en el hallazgo patognomónico presente en las 3 formas del síndrome de Currarino.

Los rayos X del colon por enema pueden orientar en presencia de masa presacra y de estenosis ano-rectal. La ultrasonografía, la tomografía y la resonanacia magnética juegan un papel importante en la delimitación de la extensión y características de la masa presacra y en el diagnóstico de otras malformaciones asociadas ${ }^{4}$. 
La biopsia rectal tiene indicaciones en los pacientes con estreñimiento para el estudio de enfermedad de Hirschpprung o presencia de disganglionosis asociada.

Actualmente no existe en la literatura un consenso establecido para el manejo terapéutico de estos enfermos pues la mayoría de informes corresponden a pequeñas series de casos con pocos individuos, sin embargo es claro que requieren una evaluación y conducta de índole multidisciplinarias.

Las propuestas de tratamiento varían entre los grupos que han hecho los informes de los casos, sin embargo, hay aspectos comunes como corregir la MAR si está presente (y según el caso se individualiza la conveniencia de una colostomía previa a la cirugía correctiva), la resección del segmento afectado si se demuestra disganglionosis o enfermedad de Hirschprung y la resección de los teratomas y las otras neoplasias por el riesgo potencial de malignización, además se debe hacer un manejo correcto de las malformaciones y las complicaciones asociadas.

En cuanto al tratamiento de los pacientes con meningocele anterior, algunos grupos proponen la corrección en todos los casos por el riesgo de complicaciones mecánicas ante el efecto de masa local sobre la vejiga o el colon o la posibilidad de meningitis por enteropatógenos, sin embargo, otros grupos prefieren un manejo conservador en los pacientes asintomáticos debido a los riesgos potenciales de daño neurológico, fístula de líquido cefalo-raquídeo y neuro-infección asociados con el procedimiento correctivo y aconsejan la cirugía sólo en casos de infección del sistema nervioso central, compresión mecánica sintomática de estructuras adyacentes o crecimiento progresivo delmeningocele. La indicación de resección del meningocele anterior en mujeres jóvenes para prevenir posibles complicaciones obstétricas futuras también se contempla en la literatu$\mathrm{ra}^{5}$.
El manejo del estreñimiento, que en la mayoría delos casos es intratable, requiere un tratamiento con laxantes en forma permanente asociado con una dieta alta en fibra.

\section{CONCLUSIÓN}

Aunque el síndrome de Currarino se describió hace más de 20 años aún se desconoce mucho de él y el subdiagnóstico en las formas leves o mínimas debe ser alto. La complejidad del síndrome requiere un enfoque multidisciplinario y es importante reconocer al paciente que se presenta con un estreñimiento crónico intratable y pensar en anomalías congénitas, que aunque poco frecuentes, pueden ser causa de un estreñimiento de origen orgánico. El tratamiento se debe individualizar según las características de cada paciente y se debe realizar estudio genético en él y en sus familiares. Se trata de un informe de caso de una entidad rara, pero al mismo tiempo de fácil diagnóstico, motivo por el cual se justifica su difusión que apoyará la literatura nacional e internacional para su conocimiento.

\section{REFERENCIAS}

1. Currarino G, Coln D, Votteler T. Triad of anorectal, sacral and presacral anomalies. Am J Roentgenol. 1981; 137: 395-8.

2. Köchling J, Karbasiyan M. Spectrum of mutations and genotype-phenotype analysis in Currarino syndrome. Eur $J$ Hum Genet. 2001; 9: 599-605.

3. Martucciello G, Torrea M. Currarino syndrome: Proposal of a diagnostic and therapeutic protocol. J Pediatr Surg. 2004; 39:1305-11.

4. Pfluger T, Czekalla R, Koletzko S, Munsterer O, Willemsen UF, Hahn K. MRI and radiographic findings in Currarino's triad. Pediatr Radiol. 1996; 26: 524-7.

5. Crétolle C, Zérah M, Jaubert F, Sarnacki S, Révillon Y, Lyonnet $\mathrm{S}$, et al. New clinical and therapeutic perspectives in Currarino syndrome (study of 29 cases). J Pediatr Surg. 2006; 41: 12631. 